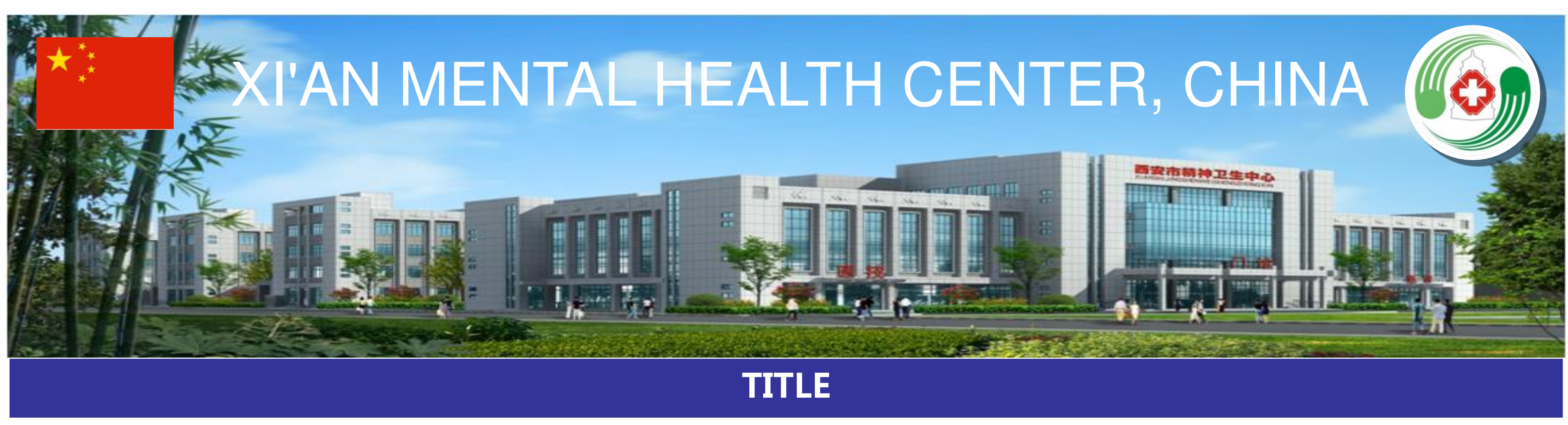

\title{
Effect of pharmacodynamics gene polymorphism on Quetiapine
}

\section{Zhang Yan*, Cui Xiaohua, Zhang xin, Feihu Liu, Wei Quan, Hui Wang, Xiaohong Zhang, Zunxiao Dai, Bin Wu, and Qiangju Wu}

Pharmaceutical laboratory, Xi'an mental health center, Xi'an, Shaanxi, China.

*The corresponding author: Yan Zhang, Xi'an Mental Health center, The eastern section of Hangtian

street, Xi'an 710100, Shaanxi, China. E-mail: zhan_777@163.com.Tel:+86-029-63609232.

\section{INTRODUCTION}

With the development of pharmacogenomics in recent years, more and more attention has been paid to the molecular genetic mechanism of pharmacokinetics among different individuals. Gene polymorphisms of drug metabolizing enzymes can affect the retention of drugs in vivo. However, the cost of gene polymorphism of drug metabolizing enzymes determination is high.

\section{OBJECTIVES}

Pharmacokinetics gene polymorphism is the important factor that affect antischizophrenia drug clinical response, study individual gene polymorphism and the connection with the efficacy of quetiapine, to select individualized medication for treatment and prevent the occurrence of adverse reactions to provide reference to quetiapine in clinic, promote the development of antischizophrenia drug individualized medication.

\section{CONCLUSION}

Patients' pharmacokinetics gene polymorphism is the important factor that affect quetiapine clinical response. 400mg is best highest dose for patients of northwest in China.

\section{METHODS}

245 patients who of collected 844 patients treated with quetiapine in Xi 'an mental health center were retrospectively analyzed, who had detected blood drug concentration and gene analysis. Analysis the relationship between the blood quetiapine concentration with CYP2D6 and CYP3A5 gene polymorphism, also evaluation quetiapine efficacy in different pharmacokinetics gene polymorphism.

\section{RESULTS}

The therapeutic efficiency in quetiapine of patients in CYP2D6 poor metabolic type (PM) was better than that of extensive metabolic type (EM), while that the therapeutic efficiency in quetiapine of CYP3A5 gene EM was higher than that of PM. The maximum dose of quetiapine in long term use should not exceed 400mg toward patients of northwest in China. 